\title{
Concept of an ecologically balanced area based on Ecological Footprint
}

\author{
H. Chen, S. Ise \& M. Taniguchi \\ Graduate School of Systems and Information Engineering, \\ University of Tsukuba, Japan
}

\begin{abstract}
s
As environmental problems increase on a global scale, a framework must be established to achieve environmental balance. Because the power of local governments is rising as a result of decentralization in Japan, local governments must shoulder the responsibility of dealing with environmental consumption within a region and set up a suitable system for an area. This study examines an ecologically balanced area based on the balance of environmental productivity and consumption capacity. Using a case study approach examining Ibaraki prefecture in Japan, which has been influenced strongly by Tokyo because of its location in the national capital region, it is possible to explain how the concept promotes understanding of ecological productivity and consumption. The study evaluated the Ecological Footprint (EF) of all 44 municipalities in Ibaraki prefecture and connected municipalities with a high environmental load with a low environmental load as one area. To the lower one, the higher one pays for its obligation for environmental management. Results of a case study demonstrated a method to let the designed areas manage their environment voluntarily and to promote local production for local consumption through interregional cooperation. This concept used in environmental planning will improve the ecological balance. The conclusions show that most municipalities in Ibaraki prefecture are not ecologically balanced and that the environmental consumption of the area is influenced by the positional relation with Tokyo metropolitan district. The median value of environmental load excess ratio of all municipalities was designed as a reduction goal in ecologically balanced areas. Consequently, the 44 municipalities in Ibaraki prefecture were formed into 11 areas, with four municipalities left without matching with the others.

Keywords: Ecological Footprint, ecologically balanced area, urban planning.
\end{abstract}




\section{Introduction}

At the Rio Summit held in 1992, many countries participated to discuss issues related to the environment. In 2012, the United Nations Conference on Sustainable Development (Rio+20) was also held in Rio as a 20-year follow-up to the 1992 Rio Summit. However, 20 years after the Rio Earth summit, the environment of the planet is becoming worse, not better, according to a report from WWF [1].

For solving environmental issues, a framework should be established. Administrative power tends to be transferred from the central bureaucracy to local governments in Japan. Through decentralization, the power of local governments is rising and local governments must shoulder the responsibility to address environmental problems. Therefore, to achieve consumption within a region, it is necessary to propose a suitable concept and to set up a suitable system considering environment balance.

This report of our study proposed an ecologically balanced area based on an Ecological Footprint, which quantifies the productive environment and that of consumption. The paper describes a case study, choosing Ibaraki prefecture in Japan to reveal the applicability of the concept for other regions. Moreover, this study presents more features of interest and of greater possibility, such as changes of the area's shape or trading system inside the area.

\section{Previous study}

This study proposed a concept of ecologically balanced area using the EF indicator as an evaluation of environmental balance. The EF indicator represents the amount of biologically productive land necessary to supply the resources a human population consumes, and to assimilate associated waste. The EF indicator was developed by Wackernagel and Rees in the early 1990s [2], and applied by many researchers. The indicator has been calculated for 150 countries at the national level by WWF [3], and cities all over the world at a regional level $[4,5]$. The EF indicator has been adopted as a proactive approach in England, such as for the environmental evaluation of project [6]. In Japan, the Ministry of the Environment provides the EF indicator as an assessment of progresses in the Basic Environmental Plan. The governments also introduce the indicator in the Regional Environmental Plan [7]. Application of the indicator has been limited to environmental evaluation or formulation of policies.

Furthermore, the EF applications in the literature are growing in number and diversity. Recently, land-use policies, specifically related to sustainable land resources, and a cap and trade systems using EF have been explored [8]. Even though studies about integrating urban planning with EF are insufficient, these studies have provided accumulations for the realization of ecologically balanced areas. 


\section{Methods}

\subsection{Calculation of EF}

This study calculates the EF Value using the Ujihara Taniguchi Model 2010.3 (EF-Calc), developed by Ujihara (Okayama University) and Taniguchi [9]. The residents' consumption is calculated using this tool based on the environmental load generated during the daily life activities of residents. Data used in the Ujihara Taniguchi Model are all publicly available and easily obtainable. Consequently, the environment load generated by urban activity outside such region, such as the industrial and service-related load, is not included.

Table 1: $\quad$ Method of EF indicator value calculation.

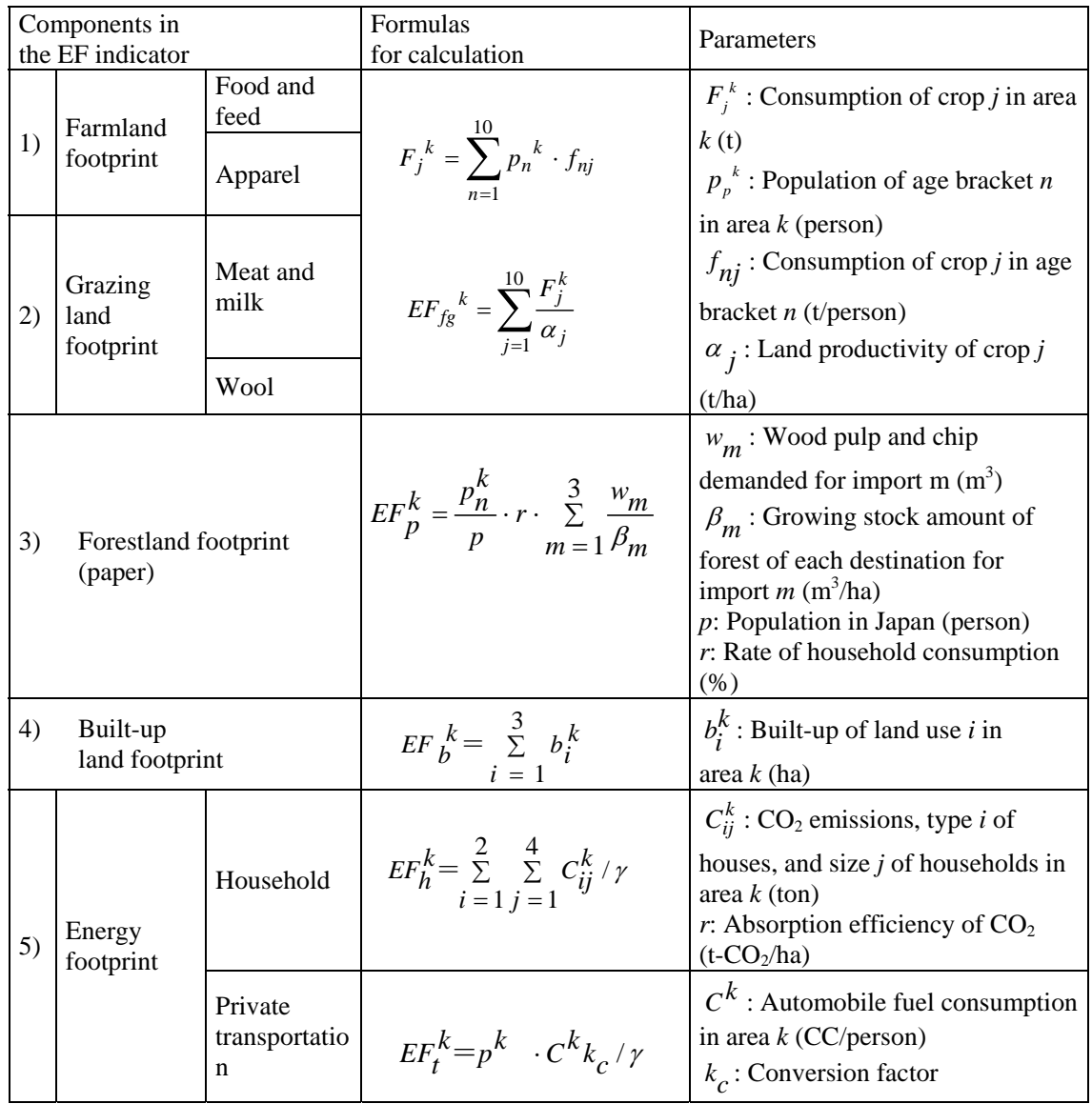

The EF indicator comprises the following components, which reference to Table 1. These components are referred from the compound EF methodology developed by Wackernagel and Rees [2]. 
1) Farmland needed to grow crops for food and feed (Farmland footprint).

2) Grazing land needed to graze animals for meat and milk (Grazing land footprint).

3) Forestland needed to obtain materials for use in paper production (Forestland footprint).

4) Built-up land needed to conduct urban activities (Built-up land footprint)

5) Forestland needed to absorb $\mathrm{CO}_{2}$ from fossil fuels for household and private transport use (Energy footprint).

Biocapacity (BC) refers to the capacity of an area to provide resources and absorb wastes of each component in EF (such as productive land for farmland).

Moreover, $r$ is defined as the environmental load excess ratio based on EF value association with residents' consumption, with reference to Table 1 . The environment balance in each region is evaluated using $r$. The environmental load excess ratio in region " $k$ " $\left(r{ }^{k}\right)$ is defined as follows.

$E F^{k}$ : EF in region " $k$ " (ha)

$$
r^{k}=\frac{E F^{k}}{B C^{k}}
$$

$B C^{k}$ : Biocapacity in region " $k$ " (ha)

\subsection{Concept of ecologically balanced area}

The ecologically balanced area proposed in this paper is a combination of a municipality with a high environmental load with that imposing a low environmental load as one area. Therefore, the dependence relation of environmental consumption is definite in terms of its area. The concept promotes the municipality with a high environmental load (consuming-municipality) to takes or pays the obligation of management by achieving environmental balance $(r \leq 1)$. The consuming municipalities shoulder the responsibility to take an approach to a suitable system for the area.

\section{Case study}

\subsection{Target region and EF value}

This case study evaluated the Ecological Footprint (EF) of all 44 municipalities in Ibaraki prefecture to review the applicability of the ecologically balanced area for other areas.

Because the concept is assumed to be applicable to the entire country, the case study ought to select a widely diverse target region covering diversified municipalities to the greatest extent possible. Therefore, this case study selected Ibaraki prefecture with its different environmental balance such as small cities and big cities. Ibaraki, which has a population of 2.96 million, is located in the outer edge of Tokyo metropolitan area, stretching eastward to the Pacific Ocean. 


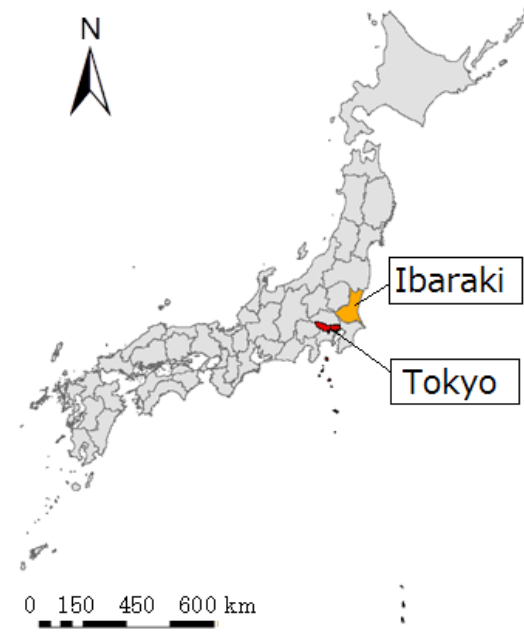

Figure 1: Location of Ibaraki in Japan.

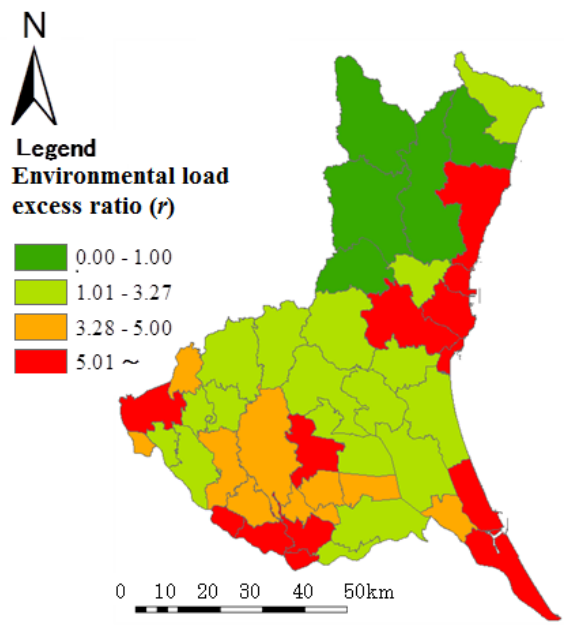

Figure 2: $\quad r$ values of 44 municipalities in Ibaraki.

The northernmost part of the prefecture is mountainous, whereas the southern part is within subway commuting distance of the capital region. Figure 1 presents the target region location.

The condition of environmental balance $(r)$ in each municipality is calculated based on the EF value associated with residents' consumption, which is given in reference to Table 1 .

Results shown in Figure 2 explained that the southern part of Ibaraki has a higher $r$, although the northern part had a lower one. This outcome occurred by reason that there is large acreage of forest to absorb wastes in the northern part. Furthermore, the urban development of the railroad line in the south caused a decrease of $\mathrm{BC}$ value and population concentration. In addition, the EF value is increased because of the concentration. This result certifies the applicability of choosing Ibaraki as a case study.

The medium environmental load excess ratio of Ibaraki is 3.27 . Only 5 municipalities among all 44 municipalities are ecologically balanced $(r \leq 1)$. Therefore, the design of an ecologically balanced area is difficult in Ibaraki prefecture.

\subsection{Design of ecologically balanced area}

Understanding of the ecologically balanced area is necessary to seek ways to design an area. Therefore, Tsukuba city is an example illustrating how to shape the area by one condition. The target city of Tsukuba, with 200 thousand people, has the largest population in Ibaraki prefecture. Figure 3 presents the location of Tsukuba city. Tsukuba developed rapidly as a result of development along the railway line, which attracted numerous commuters in the Tokyo metropolitan. 


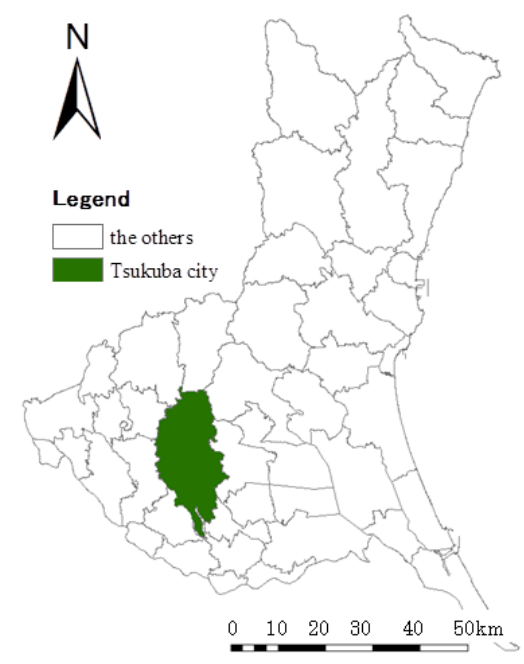

Figure 3: Tsukuba city.

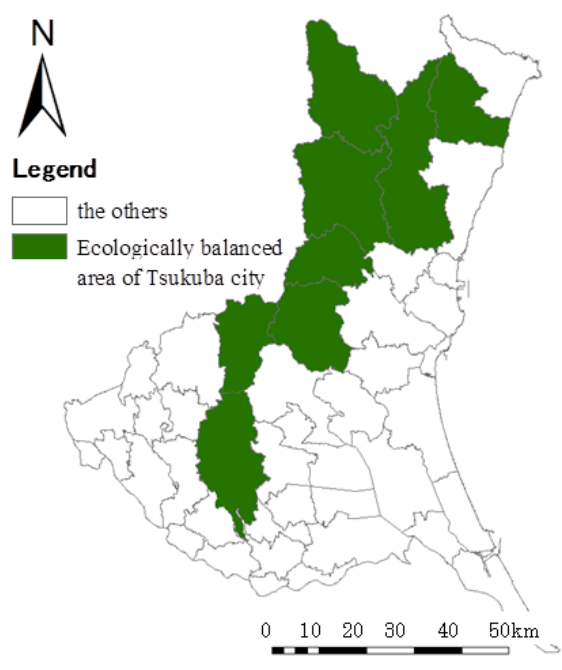

Figure 4: Example of Tsukuba's ecologically balanced area.

This study sets a condition of connecting a consuming municipality and municipalities with a low environmental load $(\alpha \leq 3.27)$ that reduces the $r$ value mostly. Investigation of all possible conditions is difficult. Therefore, the case study assumed one condition to determine the feasibility. The most ecologically balanced area of Tsukuba city designed by this condition is shown in Figure 4.

However, the area expanding from Tsukuba city to the northeast and formed a bizarre shape just like a Gerrymander [9]. Moreover, the environmental load of the area is 1.28. Results show that $r$ of the area cannot be decreased further, and that it is impossible to achieve ecological balance of every municipality in Ibaraki prefecture.

Therefore, the concept is conducted to set a target value $(\alpha)$ in the area to play an obligation on the consuming-municipalities. Several areas can be designed under the target value.

The target value $(\alpha)$ is a reference to the medium or median value. This case study set the medium value as ' $\alpha$ ', considering the extremely high environmental load in each municipality.

\subsection{Result}

As discussed in chapter 4.2, the area of Tsukuba was designed. In this chapter, all 22 consuming municipalities in Ibaraki are designed simultaneously. The 22 consuming municipalities shown in Figure 5 have environmental loads that are greater than the target value $(\alpha=3.27)$. When matching with the consuming municipality, competition occurred in several municipalities with a low environmental load. In such cases, the consuming municipality matching the municipality that reduces the $r$ value most. Results are shown in Figure 6. 


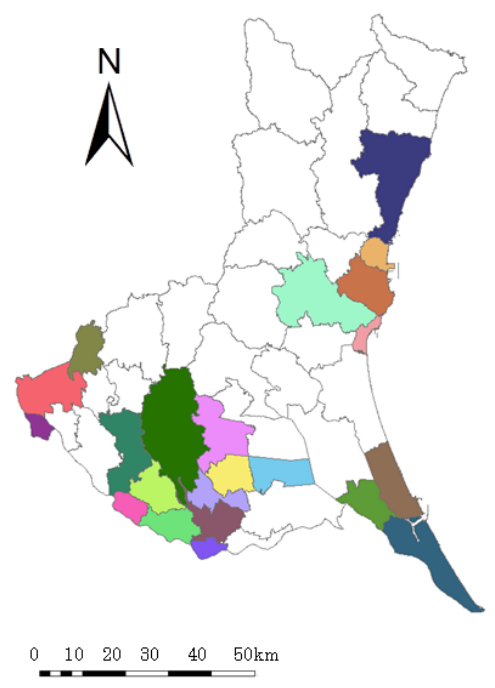

Figure 5: Municipalities in Ibaraki Figure 6: Example of ecologically with $\alpha \geqslant 3.27$.

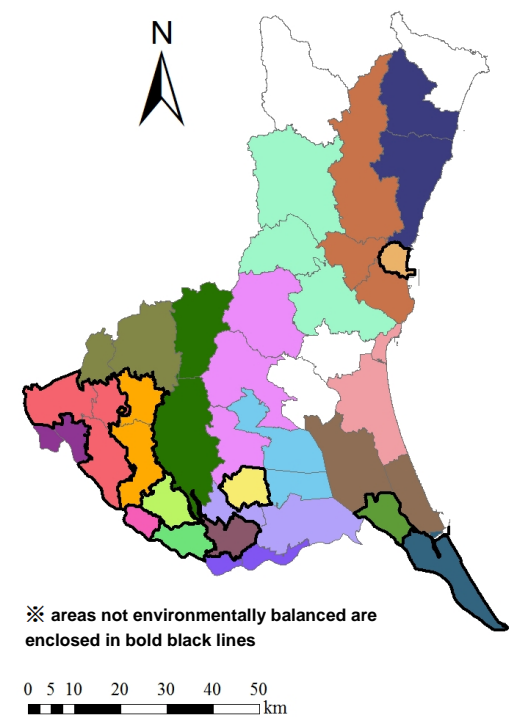

balanced area in Abaraki.

Conclusions from these analyses show that the 44 municipalities in Ibaraki prefecture were formed into 14 areas, which included 32 municipalities. In addition, 11 municipalities were left without matching with the others, and 2 areas failed to be environmentally balanced $(r \geq \alpha)$. These municipalities and areas, which are not environmentally balanced, are shown as surrounded by a bold black line in Figure 6 . However, all municipalities can be designed into ecologically balanced areas using a flexible target value $(\alpha)$. The conclusion from this analysis is that districts such as Tokyo, with a high environmental load excess ratio, can apply the concept of ecologically balanced areas.

\section{Discussion and conclusions}

The concept of ecologically balanced area based on EF is expected to become a tool promoting environmental management in an area. This study proposed a tool to clarify the amount of environmental consumption and where it has been consumed by designing areas. Based on this tool, the concept played an obligation of environment management on the consuming municipalities by setting a target value $(\alpha)$ in the area. The study demonstrated the possibility that, even if most municipalities are not ecologically balanced, areas should be made by adjusting the target value.

However, this study merely proposed a method to design an ecologically balanced area and gave no consideration to the incentives of environmental management. A system to promote voluntary measurement intended for improvement of environment balance in the area should be considered in the 
future. In fact, an interregional cap and trade system using EF was developed by Ujihara and Tanaguchi [8]. A possible exploration is to combine an ecologically balanced area with the interregional cap and trade system. This structure can contribute to improvement of the environment balance in areas from the perspective of financial resources.

Moreover, environmental management inside the areas should be explored because the concept is established to achieve an environmental balance. Such an approach is applicable to studies of individual behaviors that interact and impact the environment to benefit environment management.

\section{References}

[1] WWF, Living planet report 2012, http: //awsassets.panda.org/downloads/ 1pr_2012_rio_summary_booklet_final_120509.pdf (accessed on December 17, 2012).

[2] Wackernagel, M. \& Rees, W. E., Our Ecological Footprint: Reducing Human Impact on the Earth, New Society Publishers (Canada), 1996.

[3] WWF, Living Planet Report 2008, http: //www.wwf.or.jp/activity/lib/lpr/

[4] wwf_lpr_2008.pdf (accessed on December 17, 2012).

[5] Best Foot Forward, City Limits A resource flow and ecological footprint analysis of Greater London, 2004.

[6] Regional Progress, http: //www.regionalprogress.org/index.html, accessed on December 17, 2012.

[7] Desai, P. and Riddlestone, S., Bioregional Solutions: For living on one planet, Greenbooks, 2002.

[8] Okayama prefecture, New Okayama Basic Environmental Plan-Ecovision 2020, http://www.pref/okayama.jp/seikatsu/kansei/iso/ecovision2020.pdf, (accessed on December 17, 2012, in Japanese).

[9] Ujihara, T. and Taniguchi, M., Trading system of environmental loads: interregional cap and trade system using an ecological footprint, Ed. by Brebbia, C.A. and Beriatos, E., Sustainable development and planning V, pp. 381-396, WIT Press, 2011.

[10] Hale, N., Benjamin and Russell, J., Federalist Newspaper, 1812. 